\title{
Resonant coupling of a SQUID to a mechanical resonator
}

\author{
S. Pugnetti ${ }^{1(a)}$, Y. M. Blanter ${ }^{2}$ and R. Fazio ${ }^{1}$ \\ ${ }^{1}$ NEST, Scuola Normale Superiore \&6 CNR-INFM - Piazza dei Cavalieri 7, I-56126 Pisa, Italy, EU \\ ${ }^{2}$ Kavli Institute of Nanoscience, Delft University of Technology - Lorentzweg 1, 2628 CJ Delft, The Netherlands, EU
}

received 14 January 2010; accepted in final form 20 May 2010

published online 17 June 2010

PACS 85.25.Dq - Superconducting quantum interference devices (SQUIDs)

PACS 74.50. $+r-$ Tunneling phenomena; Josephson effects

PACS 85.85.+j - Micro- and nano-electromechanical systems (MEMS/NEMS) and devices

\begin{abstract}
We analyze the properties of a mechanical resonator embedded into a quantum SQUID and analyze under which conditions it is possible to realize a resonant coupling between the SQUID and the resonator. We find, within the present technology, how it is possible to tune the system into the regime where the plasma frequency of the SQUID matches the resonator frequency and maximizes the corresponding coupling. In these conditions the doubly degenerate quantum level of the system is split by the coupling between the SQUID and the resonator.
\end{abstract}

Copyright (C) EPLA, 2010

The interest in nanoelectromechanical systems (NEMS) has been growing rapidly $[1,2]$ because of their wide range of potential technological applications in detection and sensing, and their importance in testing fundamentals of quantum theory. The observation of the quantummechanical motion of an oscillator, demonstrated only recently for the first time [3], has important implications in understanding to which extent macroscopic objects obey the laws of quantum mechanics [4]. NEMS can play also an important role in quantum computation where they have been proposed as qubits [5], memory elements [6,7], and quantum buses $[6,8]$. Coupling of nanomechanical oscillators to a qubit has been also thoroughly investigated in the literature and many schemes of this kind have been proposed with Cooper-pair boxes [9-14], Josephson junctions (phase qubits) $[6,15]$, quantum point contacts [16], and quantum dots [17]. Recently, demonstrations of the dispersive coupling of a NEMS to a Cooper-pair box [18] and to a phase qubit [3] have been reported.

Coupling of nanomechanical oscillators to SQUIDs has been recently intensively investigated [19-24]. The high sensitivity of SQUIDs to tiny changes in the magnetic flux has suggested that the position of a nanomechanical resonator could be monitored by integrating the oscillator into the superconducting loop of a dc SQUID; indeed the transport properties of this superconducting circuit in presence of a uniform magnetic field depend on the position of the oscillator, since this position modifies the total area threaded by the flux. Recently this scheme has

\footnotetext{
(a) E-mail: s.pugnetti@sns . it
}

been demonstrated for the detection of the thermal motion of a mechanical resonator in the classical regime [25]. Due to the high degree of control achieved on quantum SQUIDs, coupling nanomechanical resonators to SQUIDs is a promising scheme for observing quantum effects in the motion of these oscillators as well.

A natural way to detect the quantum motion of the mechanical resonator would be by a spectroscopy measurement of the energy level spectrum by standard techniques developed for flux qubits [26]. Indeed the coupling of a few-level quantum system (the SQUID) with an external field can show up in the spectrum in different ways depending on whether the external field is classical or quantum $[27,28]$. In any case it is crucial that the resonant coupling regime is realized in order to produce more visible effects in a spectroscopic measurement. In this paper we show that this is however possible for a quantum SQUID coupled to a nanomechanical resonator, by tuning the external magnetic field and the bias current with available experimental setups. We discuss a strategy to optimize the resonant coupling between the resonator and the SQUID thus maximizing the splitting of an energy level associated with the SQUID due to the coupling to a resonator.

The system we consider is schematically drawn in fig. 1. A dc SQUID is made of a superconducting loop of total area $A$ with an arm of length $l$ that can oscillate freely in the plane of the loop. For simplicity we assume that only a single mode of oscillation with the frequency $\omega$ can be excited. This mode is described by the dynamical variable $X$ representing the shift of the center of mass 


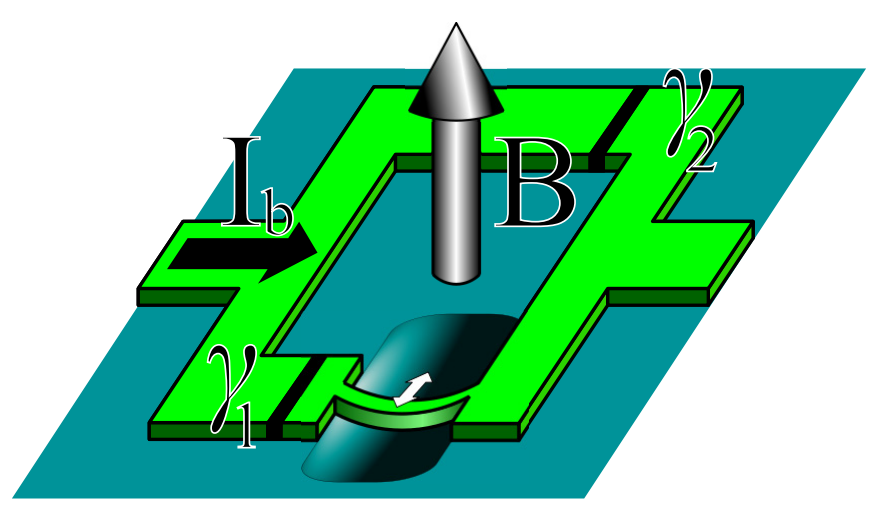

Fig. 1: (Colour on-line) A sketch of the device we study: one arm of a dc SQUID is free to oscillate in the plane of the SQUID itself. A uniform magnetic field $B$ orthogonal to the SQUID plane is present and a dc bias current $I_{b}$ flows through the device. The two Josephson junctions, whose phase drops are, respectively, $\gamma_{1}$ and $\gamma_{2}$, are taken to be identical.

of the resonator (with the mass $m$ ) with respect to its rest position. The quantum effects related to mechanical motion of this oscillator appear at the scale of the amplitude of zero-point motion $X_{0}=\sqrt{\hbar / 2 m \omega}$. The SQUID further comprises two Josephson junctions of equal critical currents $I_{c}$ and shunting capacitances $C$; the typical energy scales related to the physics of the junctions are the Josephson energy $E_{J}=\hbar I_{c} / 2 e$ and the charging energy $E_{c}=(2 e)^{2} / 2 C \ll E_{J}$, whose magnitude depends on the geometry of the junctions. The typical time scale for the dynamics of the junctions is set by the inverse plasma frequency $\omega_{\mathrm{pl}}=\sqrt{2 E_{J} E_{c}} / \hbar$. The dynamics of the SQUID is described by the two gauge-invariant phase drops $\gamma_{1}$ and $\gamma_{2}$ across the two junctions.

The coupling of the SQUID dynamics to mechanical motion is provided by the position dependence of the magnetic flux threading the SQUID loop. The two phases are constrained by the requirement that the superconducting order parameter is single valued,

$$
\gamma_{1}-\gamma_{2}=2 \pi\left(\frac{\Phi}{\Phi_{0}}+n\right)
$$

where $\Phi_{0}=h / 2 e$ is the flux quantum; the total flux $\Phi$ is the sum of three contributions. The first one comes from the external bias $\Phi_{e}=B A \equiv \Phi_{0} \phi_{e}$, while the second one $\Phi_{m}=B l X$ depends on the position of the mechanical resonator and provides the coupling between the mechanical resonator and the SQUID. If the circuit also has nonnegligible self-inductance $L$, the third contribution to the total flux $\Phi$ comes from the current circulating in the loop; if $I_{1}$ and $I_{2}$ are the currents flowing through each junction, the self-induced flux reads $L\left(I_{1}-I_{2}\right) / 2$. This device has three degrees of freedom; if $L=0$, then the constraint expressed by eq. (1) reduces the number of degrees of freedom from three to two. In the following, we assume that the dissipation effects are negligible.
Quantum coherence in the motion of the mechanical resonator can be detected via spectroscopic measurements on the quantum SQUID. Indeed, the energy levels associated with the SQUID degrees of freedom are shifted due to the coupling to the oscillator. However, this shift is of the second order in the coupling and for realistic experimental parameters is very small. We employ therefore below a different scheme, which provides the result which is of the first order in the coupling. One first chooses an appropriate set of values for the externally controllable quantities $I_{b}$ and $\Phi_{e}$ such that the system can be trapped in a minimum in which one of the eigenfrequencies of the electromagnetic modes is the same as the frequency of mechanical oscillations (to be referred below as degeneracy condition); then one moves slightly away from this degeneracy condition by changing the remaining external parameter. A plot of the energy levels as functions of the external parameters should display an avoided level crossing, which is a clear indication that the SQUID is coupled to a coherent quantum system.

It is useful to describe the system in terms of three dimensionless variables $\gamma=\left(\gamma_{1}+\gamma_{2}\right) / 2, \phi=\Phi / \Phi_{0}$ and $\xi=$ $B l X / \Phi_{0}$. The potential energy of the system reads

$$
\begin{aligned}
U= & E_{J}\left[-2 \cos \gamma \cos (\pi \phi)-\frac{I_{b}}{I_{c}} \gamma-\pi \frac{I_{b}}{I_{c}} \xi+\frac{\xi^{2}}{2 \mathscr{A}^{2}}+\right. \\
& \left.+\frac{2 \pi}{\beta_{L}}\left(\phi-\xi-\phi_{e}\right)^{2}\right]
\end{aligned}
$$

where the first term on the r.h.s. is the sum of the Josephson energies associated to the two junctions, the second term is the well-known tilting term of the "tiltedwashboard potential", the third term is necessary for getting the correct Lorentz force acting on the vibrating arm, the fourth term is the elastic potential energy and the last term represents the inductive energy of the loop due to the flux generated by the circulating current. We have also introduced the parameters

$$
\mathscr{A}=\sqrt{\frac{2 E_{J}}{\hbar \omega}} \frac{B l X_{0}}{\Phi_{0}} ; \quad \beta_{L}=\frac{2 L I_{c}}{\Phi_{0}} .
$$

The parameter $\mathscr{A}$ is proportional to the flux threading the area swept by the mechanical resonator and plays the role of a coupling parameter, as we show below. Typical values of $\mathscr{A}=4 \times 10^{-5}$ can be obtained assuming $I_{c}=1 \mu \mathrm{A}$, $\omega=1 \mathrm{GHz}, l=1 \mu \mathrm{m}, X_{0}=10 \mathrm{fm}$ and $B=0.1 \mathrm{~T}$. When the temperature is low enough for the system to reach the quantum regime, the coordinates typically oscillate around a minimum of the potential corresponding to the values $\bar{\gamma}$, $\bar{\phi}$ and $\bar{\xi}$. We can approximate the dynamics as a threedimensional harmonic oscillator, with the energy being a quadratic form,

$$
E_{\mathrm{tot}}=\sum_{i} \frac{\hbar^{2}}{2 E_{c}} \dot{q}_{i}^{2}+\sum_{i, j} E_{J} V_{i j} q_{i} q_{j}
$$


where the coordinates $q_{i}$ read

$$
q_{1}=\gamma-\bar{\gamma} ; \quad q_{2}=\pi(\phi-\bar{\phi}) ; \quad q_{3}=\frac{1}{\Omega} \frac{1}{\sqrt{2} \mathscr{A}}(\xi-\bar{\xi})
$$

and

$$
V=\left(\begin{array}{ccc}
r & -s & 0 \\
-s & r+\frac{2}{\pi \beta_{L}} & -\frac{2 \sqrt{2} \mathscr{A}}{\beta_{L}} \Omega \\
0 & -\frac{2 \sqrt{2} \mathscr{A}}{\beta_{L}} \Omega & \left(1+\frac{4 \pi \mathscr{A}^{2}}{\beta_{L}}\right) \Omega^{2}
\end{array}\right) .
$$

(We have introduced $r=\cos \bar{\gamma} \cos (\pi \bar{\phi}), s=\sin \bar{\gamma} \sin (\pi \bar{\phi})$ and $\left.\Omega=\omega / \omega_{\mathrm{pl}}\right)$. The coupling $V_{23}$ between the mechanical resonator and the SQUID is proportional to the ratio $\mathscr{A} / \beta_{L}$ (for devices with a low self-inductance this behavior no longer holds, see below for discussion); the decoupled regime can be obtained for either $B \rightarrow 0$ or $L \rightarrow \infty$. The coordinate $q_{1}=\gamma-\bar{\gamma}$, corresponding to one of the electromagnetic degrees of freedom, oscillates with the frequency $\omega_{\mathrm{pl}} \sqrt{\cos \bar{\gamma} \cos (\pi \bar{\phi})}$; therefore if a minimum $(\bar{\gamma}, \bar{\phi}, \bar{\xi})$ is such that

$$
\cos \bar{\gamma} \cos (\pi \bar{\phi})=\Omega^{2}
$$

the frequencies associated with the motion of average phase drop $\gamma$ and oscillator motion coincide up to correction of second order in $\mathscr{A}$; we show below that the equality is indeed exact to all orders in the coupling. Equation (6) is exact up to quadratic terms in the displacement from the equilibrium position; however it is not suitable for describing devices with generic values of the self-inductance parameter $\beta_{L}$, since four matrix elements diverge for $\beta_{L} \rightarrow 0$. These divergences reflect the fact that for $\beta_{L}=0$ only two degrees of freedom should remain, the value of the third coordinate being fixed by the other two. A description could be obtained by switching to a basis in which the diverging subblock is diagonal; without any loss of generality, the new form is suitable for all kinds of situation, including $\beta_{L} \simeq 0$. The analytic expression for the matrix in the new basis is quite cumbersome; below we give this expression for the case in which the degeneracy condition (7) holds,

$$
V^{\prime}=\left(\begin{array}{ccc}
\Omega^{2} & -s \frac{\sqrt{2} \pi \mathscr{A}}{C} \Omega & \frac{s}{C} \\
-s \frac{\sqrt{2} \pi \mathscr{A}}{C} \Omega & \Omega^{2} & 0 \\
\frac{s}{C} & 0 & \Omega^{2}+\frac{2 C^{2}}{\pi \beta_{L}}
\end{array}\right),
$$

with $C=\sqrt{2 \pi^{2} \mathscr{A}^{2} \Omega^{2}+1}$. The first and second row and column correspond to the phase drop $\gamma$ and to the mechanical degree of freedom, respectively. The parameters $I_{b}$ and
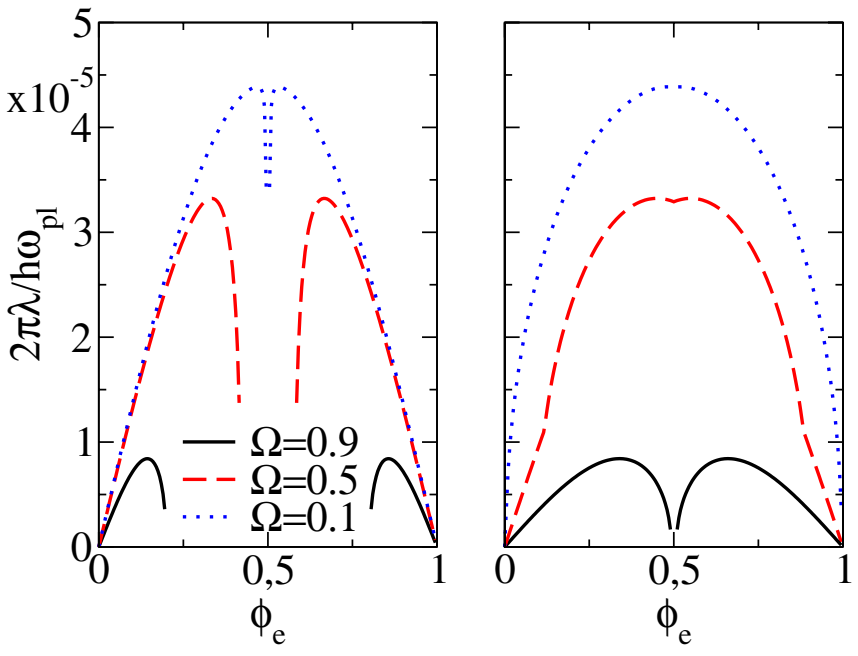

Fig. 2: (Colour on-line) Dimensionless splitting $\lambda / \hbar \omega_{\mathrm{pl}} v s$. the external flux $\phi_{e}$ for the coupling parameter $\mathscr{A}=4 \times 10^{-5}$. The self-inductance parameter $\beta_{L}$ is $10^{-4}$ for the left panel and 1 for the right panel. Dotted, dashed, and solid lines correspond to $\Omega=\omega / \omega_{\mathrm{pl}}=0.1,0.5$ and 0.9 , respectively. The plots are periodic, the period being one flux quantum.

$\Phi_{e}$ corresponding to the degeneracy condition are found if one solves the equation set,

$$
\left\{\begin{array}{l}
I_{b} / I_{c}=2 \sin \bar{\gamma} \cos (\pi \bar{\phi}) \\
2 \beta_{L}^{-1}\left(\bar{\phi}-\phi_{e}-\bar{\xi}\right)=-\cos \bar{\gamma} \sin (\pi \bar{\phi}), \\
\bar{\xi}=4 \pi \mathscr{A}^{2} \beta_{L}^{-1}\left(\bar{\phi}-\phi_{e}-\bar{\xi}+\left(\beta_{L} I_{b}\right) /\left(4 I_{c}\right)\right), \\
\cos \bar{\gamma} \cos (\pi \bar{\phi})=\Omega^{2}
\end{array}\right.
$$

where the first three lines are satisfied by stationary points of the potential energy (2) and the fourth line is the degeneracy condition. In a minimum one must have $V>0$. The unknowns are the coordinates $(\bar{\gamma}, \bar{\phi}, \bar{\xi})$ of the minimum and $\Phi_{e}$; we use $I_{b}$ to tune the system to the degeneracy point ${ }^{1}$.

Next, we quantize the system. The Hamiltonian reads

$$
H=\sum_{i=1}^{3} \hbar \omega_{i} a_{i}^{\dagger} a_{i}+\sum_{i \neq j} \frac{1}{4} \hbar \omega_{\mathrm{pl}} \frac{\omega_{\mathrm{pl}}}{\sqrt{\omega_{i} \omega_{j}}} V_{i j}^{\prime}\left(a_{i}^{\dagger}+a_{i}\right)\left(a_{j}^{\dagger}+a_{j}\right)
$$

with $\omega_{i}=\omega_{\mathrm{pl}} \sqrt{V_{i i}^{\prime}}$. When the condition (7) is fulfilled, the first excited levels $|100\rangle$ and $|010\rangle$ are quasi-degenerate and the Hamiltonian (10) restricted to their subspace becomes

$$
H=\left(\begin{array}{cc}
\hbar \omega & \lambda \\
\lambda & \hbar \omega
\end{array}\right), \quad \lambda=\frac{1}{4} \hbar \omega_{\mathrm{pl}} \frac{1}{\Omega} V_{\xi \gamma}^{\prime}
$$

Figure 2 shows the dependence of the dimensionless level splitting $\lambda / \hbar \omega_{\mathrm{pl}}$ on the dimensionless external

\footnotetext{
${ }^{1}$ In principle one, many or no solutions can exist for a particular set of parameters; when more than one solution exists, we take the one with the bigger gap $\lambda$.
} 

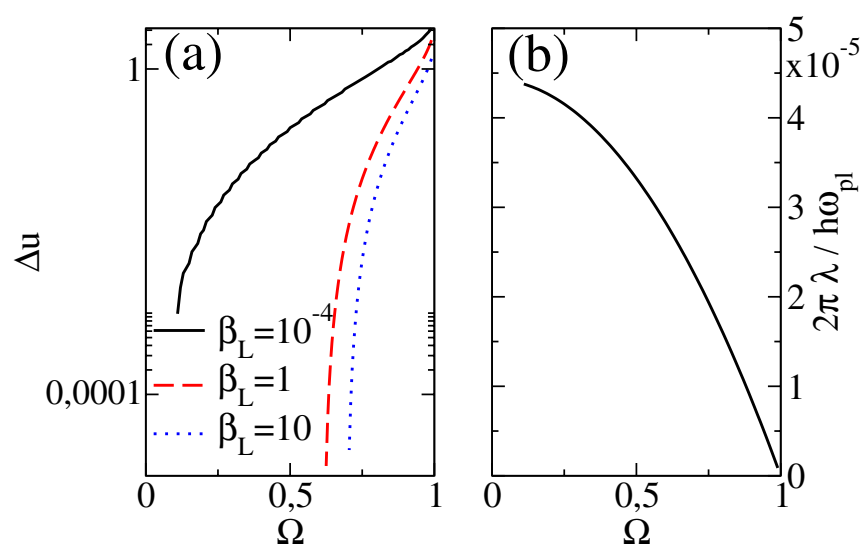

Fig. 3: (Colour on-line) (a) The dimensionless depth $\Delta u$ of the minimum featuring maximum splitting $\lambda$ is plotted as a function of the ratio $\Omega=\omega / \omega_{\mathrm{pl}}$, for different values of the selfinductance parameter: $\beta_{L}=10^{-4}$ (solid line), $\beta_{L}=1$ (dashed line) and $\beta_{L}=10$ (dotted line). (b) The maximum value of the dimensionless splitting $\lambda / \hbar \omega_{\mathrm{pl}}$ is plotted as a function of $\Omega$; here $\beta_{L}=1$, but the result is independent of $\beta_{L}$.

flux $\phi_{e}$. The maximum level splitting is obtained for a specific value of the external flux and depends very weakly on the loop self-inductance; however the value of the flux at which the maximum is achieved, does; see below for further discussion. The magnitude of the splitting is of the order of $10^{-5} \hbar \omega_{\mathrm{pl}}$. The value of the ratio $\Omega=\omega / \omega_{\mathrm{pl}}$ plays an important role: lower values correspond to bigger maximum splittings and are thus preferable.

To enable the detection, the potential well formed at the chosen minimum must be capable of containing quantum states. The number of bound states can be estimated by the ratio between the energy difference $\Delta U$ between the minimum and the closest saddle point and the energy level separation, which is roughly $\hbar \omega$,

$$
\frac{\Delta U}{\hbar \omega}=\frac{\Delta U}{E_{J}} \frac{1}{\Omega} \sqrt{\frac{E_{J}}{2 E_{c}}} \equiv \Delta u \sqrt{\frac{E_{J}}{2 E_{c}}} .
$$

Figure 3a displays plots of the quantity $\Delta u$, defined by eq. (12), as a function of $\Omega$ for different values of the self-inductance parameter $\beta_{L}$; one sees that small values of $\Omega$ correspond to very shallow minima in the potential energy, whereas the deepest minima correspond to devices in which $\omega=\omega_{\mathrm{pl}}$. However fig. 3b shows that for these devices no gap is expected in the first order in the coupling; this rather surprising fact can be further illustrated by considering the dependence of the matrix element $V_{\gamma \xi}^{\prime}$ on $s=\sin \gamma \sin (\pi \phi)$ in eq. (8): for $\Omega=1$, one has $\cos \gamma \cos (\pi \phi)=0$ and thus $s=0=\lambda$. Because of this trade-off, the optimal condition corresponds to an intermediate case. Note, however, that $\Delta u$ depends on $E_{J}$ and $E_{c}$ only via the ratios $\Omega=\omega / \omega_{\mathrm{pl}}$ and $I_{b} / I_{c}$ and thus can be tuned independently of $E_{J} / E_{c}$. Therefore a bigger minimum depth can be obtained by designing the SQUID so that the ratio $E_{J} / E_{c}$ is large.
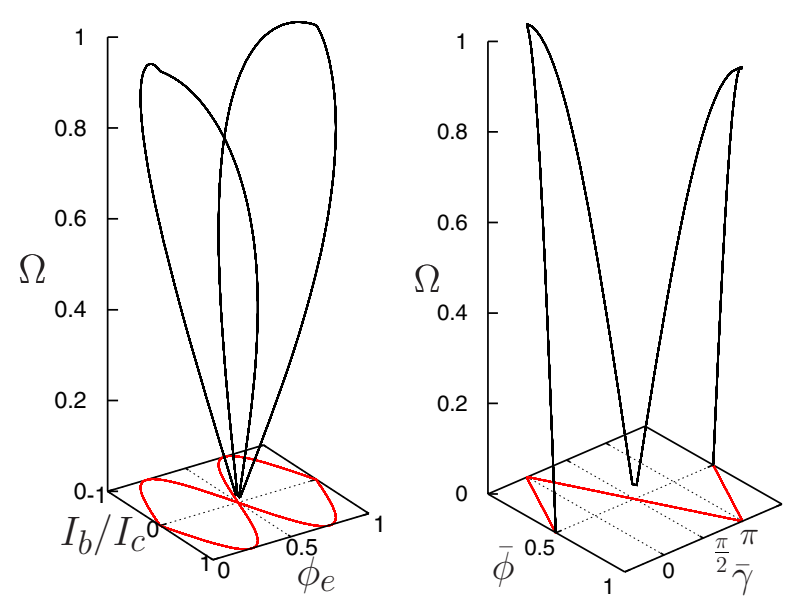

Fig. 4: (Colour on-line) Plots of the solutions of eqs. (9) giving the maximum value of the dimensionless splitting $\lambda / \hbar \omega_{\mathrm{pl}}$; left panel: $\left(I_{b} / I_{c}, \phi_{e}, \Omega\right)$, right panel: $(\bar{\phi}, \bar{\gamma}, \Omega)$. A projection of the points onto to the horizontal plane is also displayed for reader's convenience.

We now comment on the role of the self-inductance. The curve in fig. $3 \mathrm{~b}$ is very little affected by the value of $\beta_{L}$, implying that this parameter is not relevant for improving the splitting $\lambda$; on the contrary, the curves in fig. 3a show that the dimensionless depth $\Delta u$ of the minimum well is drastically reduced by increasing the self-inductance of the loop. Thus loops of smaller self-inductance are preferable.

Finally, fig. 4 represents numerical solutions of eqs. (9) used in the previous figures. The left panel shows the values of the dimensionless external parameters $I_{b} / I_{c}$ and $\phi_{e}$ that corresponds to the maximum value of the gap $\lambda$ for values of $\Omega$ between 0 and 1 , while the right panel shows a plot of the coordinates $(\bar{\gamma}, \bar{\phi})$ of the best minimum. As expected, the results are symmetric with respect to simultaneous current inversion $I_{b} \rightarrow-I_{b}$ and flux reflection $\phi_{e} \rightarrow 1-\phi_{e}$; this can be seen from eqs. (9), where in this case if $\left(\bar{\gamma}, \bar{\phi}, \bar{\xi}, I_{b} / I_{c}\right)$ is a solution, then also $\left(\pi-\bar{\gamma}, 1-\bar{\phi},-\bar{\xi},-I_{b} / I_{c}\right)$ solves the equations.

In the whole presentation we ignored the finite quality factor of the resonator and the decoherence of the SQUID. In this respect the required analysis goes exactly along the same lines as, for example, discussed in [26]. Damping should be small enough so that the line width should be small as compared with the energy splitting. The absolute value of the splitting depends on the bare plasma frequency $\omega_{\mathrm{pl}}=\sqrt{2 E_{c} E_{J}} / \hbar$ of the SQUID; for $\omega_{\mathrm{pl}} \sim 100 \mathrm{GHz}$, the splitting $2 \lambda$ can be as large as several MHz. Therefore if both the dephasing time of the SQUID and the decay time of the mechanical oscillator exceed $1 \mu \mathrm{s}$, the avoided crossing in the spectrum should be observable; for quantum SQUIDs this has already been reported [29], whereas for $\mathrm{GHz}$ mechanical resonators it should not be a problem if using suspended carbon nanotubes as oscillators [30]. Also the spectroscopic measurement is identical to that, by now, routinely performed in SQUIDs in the quantum regime. The missing 
piece of information, provided by the present work, was to understand how to achieve the resonant coupling.

In conclusion, we found under which conditions quantum oscillations of a NEMS embedded into a SQUID can be detected by spectroscopic measurements in the regime when one of the plasma frequencies of the SQUID matches the frequency of the mechanical resonator. This frequency matching is possible with the current experimental techniques, and the scheme has two parameters (external flux and external current) to simultaneously tune the system to the vicinity of the degeneracy point and perform spectroscopic measurements around this point.

We acknowledge the financial support of the Future and Emerging Technologies programme of the European Commission, under the FET-Open project QNEMS (233992). We thank H. VAN DER ZANT, S. ETAKI, M. Poot, H. Meerwaldt and B. Schneider for useful discussions.

\section{REFERENCES}

[1] Cleland A. N., Fundations of Nanomechanics (Springer, Berlin) 2003.

[2] Blencowe M., Phys. Rep., 395 (2004) 159.

[3] O'Connell A. D., Hofheinz M., Ansmann M., Bialczak R. C., Lenander M., Lucero E., Neeley M., Sank D., Wang H., Weides M., Wenner J., Martinis J. M. and Cleland A. N., Nature, 464 (2010) 697.

[4] Leggett A. J., J. Phys.: Condens. Matter, 14 (2002) R415.

[5] Savel'ev S., Hu X. and Nori F., New J. Phys., 8 (2006) 105.

[6] Cleland A. N. and Geller M. R., Phys. Rev. Lett., 93 (2004) 070501.

[7] Pritchett E. J. and Geller M. R., Phys. Rev. A, 72 (2005) 010301.

[8] Zou X. B. and Mathis W., Phys. Lett. A, 324 (2004) 484.

[9] Armour A. D., Blencowe M. P. and Schwab K. C., Phys. Rev. Lett., 88 (2002) 148301.
[10] Martin I., Shnirman A., Tian L. and Zoller P., Phys. Rev. B, 69 (2004) 125339.

[11] Rabl P., Shnirman A. and Zoller P., Phys. Rev. B, 70 (2004) 205304.

[12] Wei L. F., Xi Liu Y., Sun C. P. and Nori F., Phys. Rev. Lett., 97 (2006) 237201.

[13] Armour A. D. and Blencowe M. P., New J. Phys., 10 (2008) 095004.

[14] Hauss J., Fedorov A., André S., Brosco V., Hutter C., Kothari R., Yeshwanth S., Shnirman A. and Schön G., New J. Phys., 10 (2008) 095018.

[15] Trees B. R., Helal Y. H., Schiffrin J. S. and Siller B. M., Phys. Rev. B, 76 (2007) 224513.

[16] Ruskov R., Schwab K. and Korotkov A. N., Phys. Rev. B, 71 (2005) 235407.

[17] Liao J. Q. and Kuang L. M., Eur. Phys. J. B, 63 (2008) 79.

[18] Lahaye M. D., Suh J., Echternach P. M., Schwab K. C. and Roukes M. L., Nature, 459 (2009) 960.

[19] Zhou X. and Mizel A., Phys. Rev. Lett., 97 (2006) 267201.

[20] Buks E. and Blencowe M. P., Phys. Rev. B, 74 (2006) 174504.

[21] Xue F., Liu Y. X., Sun C. P. and Nori F., Phys. Rev. B, 76 (2007) 064305.

[22] Wang Y.-D., Semba K. and Yamaguchi H., New J. Phys., 10 (2008) 043015.

[23] Zhang J., Liu Y.-Xi and Nori F., Phys. Rev. A, 79 (2009) 052102.

[24] Pugnetti S., Blanter Y. M., Dolcini F. and Fazio R., Phys. Rev. B, 79 (2009) 174516.

[25] Etaki S., Poot M., Мahboob I., Onomitsu K., Yamaguchi H. and van Der Zant H. S. J., Nat. Phys., 4 (2008) 785.

[26] Mooij J. E., Orlando T. P., Levitov L., Tian L., VAN DER WAL C. H. and Lloyd S., Science, 285 (1999) 1036.

[27] Schuster I., Kubanek A., Fuhrmanek A., Puppe T., Pinkse P. W. H., Murr K. and Rempe G., Nat. Phys., 4 (2008) 382.

[28] Fink J. M., Göppl M., Baur M., Bianchetti R., Leek P. J., Blais A. and Wallraff A., Nature, 454 (2008) 315.

[29] Bertet P., Chiorescu I., Burkard G., Semba K., Harmans C. J. P. M., DiVincenzo D. P. and MooiJ J. E., Phys. Rev. Lett., 95 (2005) 257002.

[30] Meerwaldt H., private communication. 